\title{
Closed microsurgery for diabetic traction macular detachment
}

\author{
TOM BARRIE, ${ }^{*}$ ELIAS FERETIS, $\dagger$ PETER LEAVER, AND DAVID MCLEOD \\ From the Surgical Vitreoretinal Unit, Moorfields Eye Hospital, London
}

SUMMARY Results of closed microsurgery are reported in a consecutive series of 50 eyes with diabetic traction retinal detachment involving the macula. The extent of fibrovascular epiretinal proliferation profoundly influenced the outcome of surgery.

Ischaemic diabetic retinopathy may be complicated by multiple outgrowths of fibrovascular tissue on to the retinal surface within the most cortical part of the vitreous gel, typically along the major vascular arcades and nasal to the optic disc. Tangential traction, exerted by contraction of the coalescent epiretinal membranes, results in distortion, folding, and detachment of the underlying retina. This process is frequently exacerbated by traction along the detached posterior hyaloid interface between the vitreous base anteriorly and the epiretinal membranes into which the gel is incarcerated posteriorly (anteroposterior traction) or between individual sites of fibrovascular proliferation (bridging traction). A retinal break, usually sited immediately peripheral to the avulsed edge of a posterior fibrovascular membrane, may also contribute to retinal separation by allowing exchange of fluid between the retrohyaloid and subretinal spaces.

The fovea, which is usually free of overlying vasoproliferation, may eventually become detached, with consequent serious impairment of vision. Closed microsurgical techniques can be used to eliminate each of the elements of vitreoretinal traction described above and to seal retinal breaks, thus allowing the macular retina to reattach with some restoration of central visual function. We present the results of closed microsurgery performed for diabetic traction macular detachment in the Surgical Vitreoretinal Unit at Moorfields Eye Hospital. The surgical prognosis is correlated with the extent of preoperative epiretinal fibrovascular proliferation.

Correspondence to Mr D McLeod, FRCS, Moorfields Eye Hospital, City Road, London EC1V 2PD.

*Present address: Gartnavel General Hospital, Glasgow. tPresent address: General Hospital of Patras, Greece.

\section{Material and methods}

A consecutive series of 50 eyes from 48 diabetic patients, operated upon by 2 of us (DMcL or PL) for macular retinal detachment, were chosen for study. The surgery was performed between September 1979 and August 1981, that is to say, subsequent to the introduction of common-gauge microsurgery and scissors dissection in our hospital. The patients ranged in age from 24 to 68 years (average 45 years); there were 27 males and 21 females. All but 4 eyes were free of rubeosis iridis, which, if present, was of modest degree without secondary glaucoma. In all cases the central visual axis was sufficiently clear to allow preoperative ophthalmoscopic identification of macular elevation, so that visual improvement after surgery related to macular reattachment and not to removal of opacities in the media. The detachment was of 'pure traction' type in 26 eyes, and 'combined rhegmatogenous and traction' type in 24 eyes.

The detachments were graded preoperatively according to the extent of fibrovascular epiretinal proliferation (Table 1; Figs. 1A, B, C: Fig. 1 shows fundus photographs of representative fields of 4 right eyes). The extent of fibrovascular tissue avulsed from the retinal surface or proliferating along the detached posterior hyaloid interface was not taken into consideration. Eyes in grade 1 (with less than 4 disc diameters of proliferation) either had membranes located very close to the macula or were of the combined traction and rhegmatogenous type (Fig. 1A). We surmised that, in view of the location and limited extent of proliferation, the latter eyes would not have proceeded to macular detachment had a retinal break not developed. Eyes in grade 3, with more than 6 disc diameters of proliferation (Fig. 1C), frequently showed nonvascularised epimacular pro- 


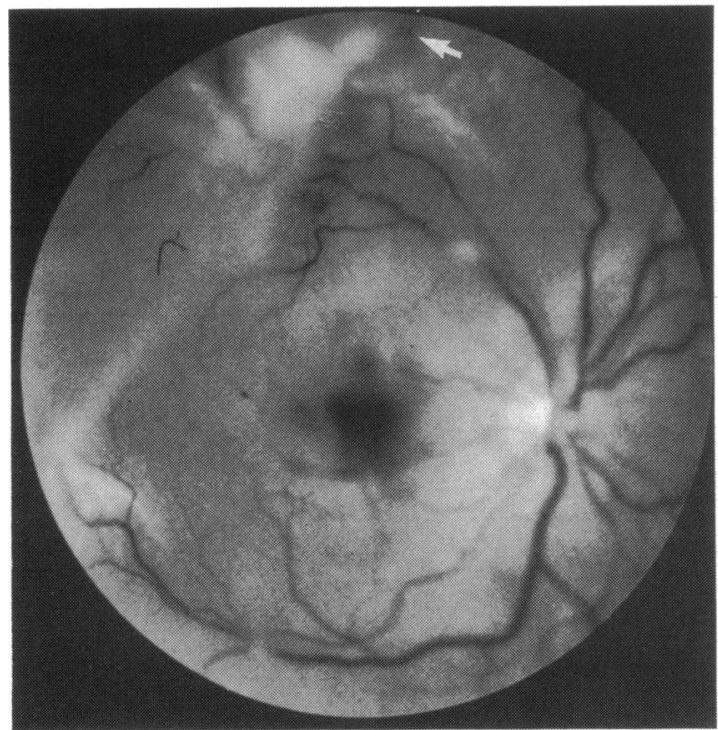

Fig. 1A Combined traction and rhegmatogenous detachment with grade 1 proliferation; one of 2 retinal breaks arrowed; subfoveal haemorrhage.

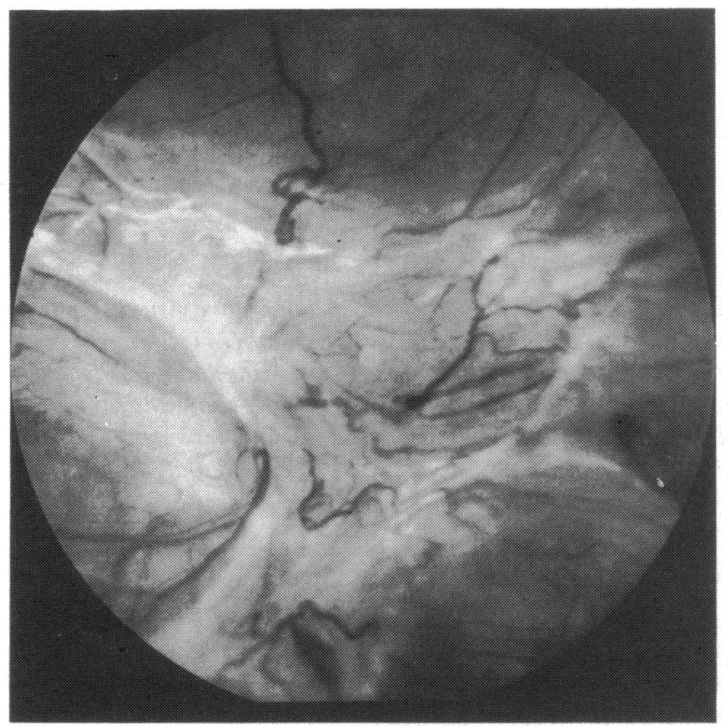

Fig. 1C Peripapillary region of eye with grade 3 proliferation and traction detachment.

liferation. In some eyes, the posterior retina was completely covered by coalescent fibrovascular membranes with variable retinal elevation towards a table-top configuration of detachment. Several eyes with grade 3 proliferation, often producing a tabletop detachment, were treated by closed microsurgery and a fluid/liquid silicone exchange ${ }^{1}$ during the period

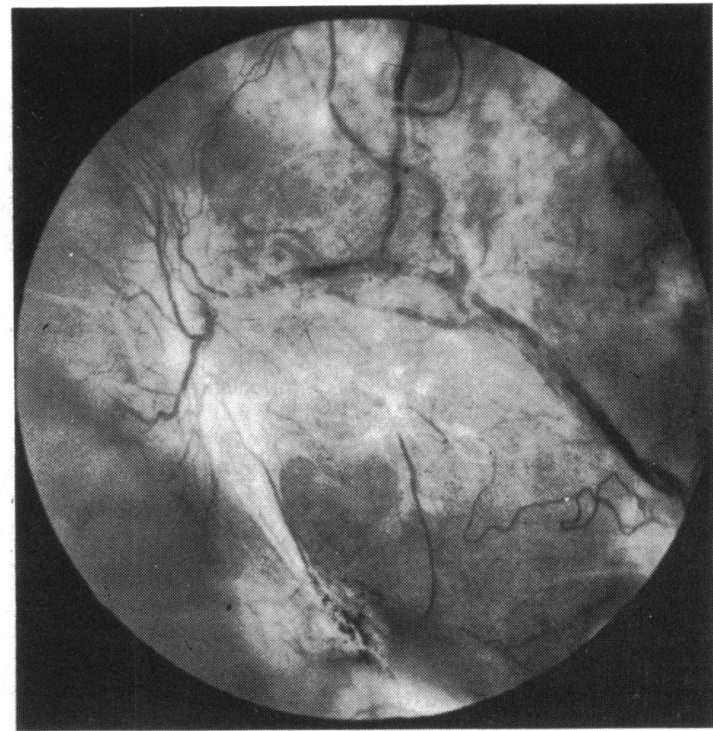

Fig. 1B Superior macular region of eye with grade 2 proliferation and traction detachment.

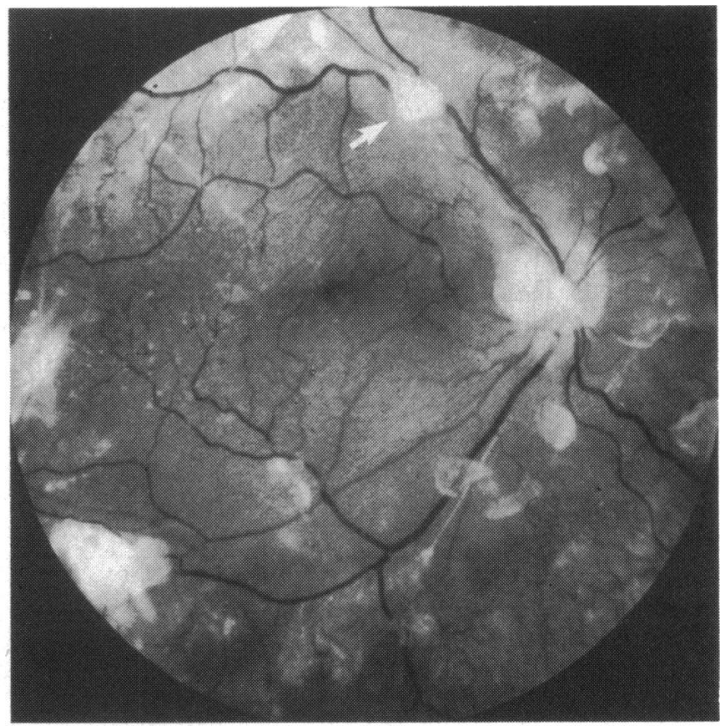

Fig. 1D Postoperative picture of eye which had grade 3 proliferation preoperatively; arrow points to one of 18 segmented fibrovascular outgrowths in photographic field.

in question; such eyes have been excluded from consideration. The reported series represents approximately $30 \%$ of all diabetic vitrectomies performed in our unit during this period.

Common-gauge microsurgery was carried out via 3 pars plana sclerotomies in the fashion popularised by Charles. ${ }^{2}$ The vitreous gel and posterior hyaloid 
Table 1 Grading of eyes

\begin{tabular}{|c|c|c|c|c|}
\hline $\begin{array}{l}\text { Grade 1: } \\
\text { Grade 2: } \\
\text { Grade 3: }\end{array}$ & \multicolumn{3}{|c|}{$\begin{array}{l}<4 \text { disc diameters of fibrovascular epiretinal } \\
\text { proliferation } \\
4-6 \text { disc diameters of fibrovascular epiretinal } \\
\text { proliferation } \\
>6 \text { disc diameters of fibrovascular epiretinal } \\
\text { proliferation }\end{array}$} & $\begin{array}{l}7 \text { eyes } \\
22 \text { eyes } \\
21 \text { eyes }\end{array}$ \\
\hline Table 2 & \multicolumn{4}{|c|}{ Results of surgery } \\
\hline & $\begin{array}{l}\text { Anatomical } \\
\text { success }\end{array}$ & $\begin{array}{l}\text { Vision } \\
\text { improved }\end{array}$ & $\begin{array}{l}\text { Vision } \\
\text { unchanged }\end{array}$ & $\begin{array}{l}\text { Vision } \\
\text { worse }\end{array}$ \\
\hline Grade 1 & $7 / 7(100 \%)$ & $6 / 7(86 \%)$ & $0 / 7(0 \%)$ & $1 / 7(14 \%)$ \\
\hline Grade 2 & $20 / 22(91 \%)$ & $17 / 22(77 \%)$ & $1 / 22(5 \%)$ & $4 / 22(18 \%)$ \\
\hline Grade 3 & $10 / 21(48 \%)$ & $9 / 21(43 \%)$ & $0 / 21(0 \%)$ & $12 / 21(57 \%)$ \\
\hline All grades & $37 / 50(74 \%)$ & $32 / 50(64 \%)$ & $1 / 50(2 \%)$ & $17 / 50(34 \%)$ \\
\hline
\end{tabular}

tissue subserving anteroposterior and bridging traction were removed either with the Ocutome or with the 20-gauge Kloti vitreous stripper such that vitreous attachments to posterior epiretinal membranes were circumscised. The fibrovascular membranes were segmented (Fig. 1D) by means of manually operated vitreous scissors made by Medical Workshops (Groningen) or Osborn and Simmons (London), and the contained vessels were coagulated by bimanual bipolar diathermy. ${ }^{2}$ In those eyes with pre-existing or iatrogenic retinal breaks, subretinal fluid was drained internally by either a modified Charles flute needle ${ }^{3}$ or the Charles linear suction device. ${ }^{2}$ Simultaneous exchange of vitreal and subretinal fluid for a $20 \%$ SF6/air mixture was then performed. Retinal breaks were treated by transscleral cryotherapy, and cryotherapy was routinely performed to the immediately postoral retina in the region of the pars plana entry sites. In many cases the peripheral cryotherapy was extended through $360^{\circ}$, often together with a limited panretinal cryoablation or endophotocoagulation ${ }^{2}$ of attached retina. Thirtyseven eyes were encircled, and, if posterior retinal breaks could not be fully mobilised, they were covered by a radial sponge explant ( 4 eyes). In 33 phakic eyes there was either difficulty in peripheral dissection, a need for simultaneous fluid/gas exchange to be visualised biomicroscopically, or a problem in controlling membrane vascularity, so the lens was removed with the Keeler Phacolevigator or the Berkeley Fragmatome.

To maintain effective internal closure of breaks in those eyes containing an SF6/air mixture patients assumed an appropriate posture for several days postoperatively. A second operative procedure was performed on 8 eyes because of redetachment ( 6 eyes) or vitreous haemorrhage ( 2 eyes), 3 of which ultimately had a successful outcome. The postoperative follow-up period was 6-28 months, during

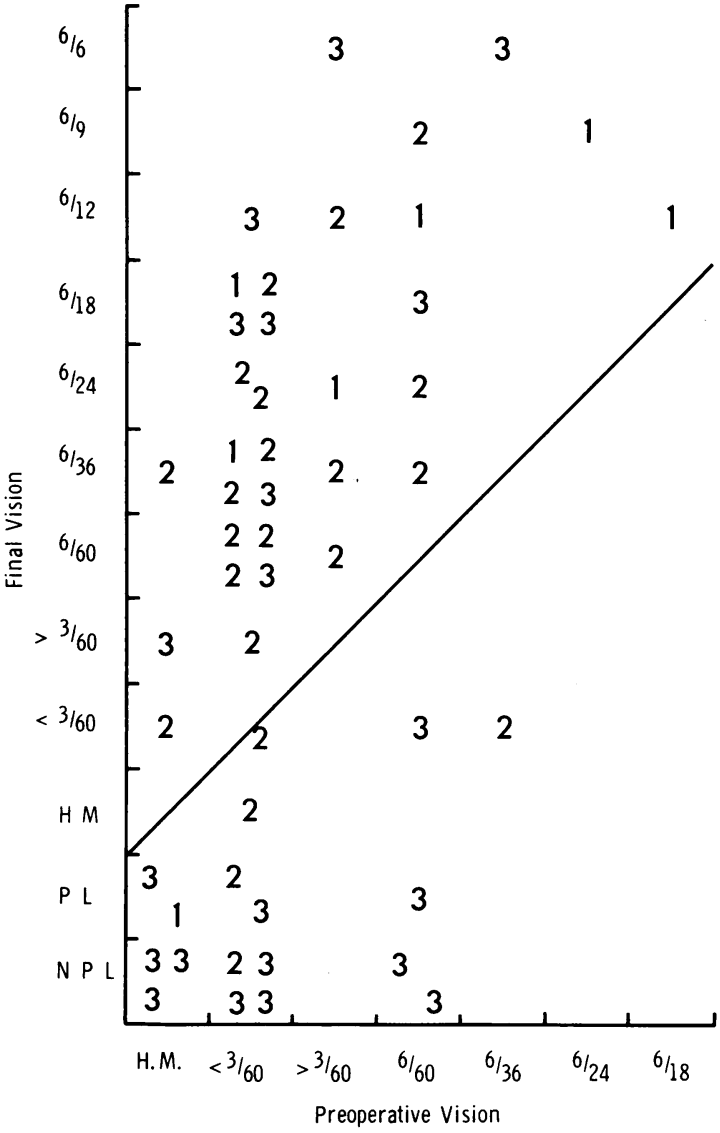

Fig. 2 Scattergram comparing preoperative vision and final vision in 50 consecutive eyes; each number $(1,2$, or 3$)$ represents an eye with grade 1, 2, or 3 epiretinal vasoproliferation respectively. Numbers above the oblique line represent eyes with improved vision after surgery. $H M=$ hand motion vision. $P L=$ perception of light. $N P L=$ no perception of light.

which interval most eyes with clear media and an attached retina underwent argon laser scatter photocoagulation, often to supplement a preoperative (14 eyes) or intraoperative ablation. No patients died within 6 months of surgery.

\section{Results}

Surgical reattachment of the macula was achieved and maintained in $74 \%$ of eyes in this series and visual improvement occurred in 64\% (Table 2). The most frequent cause of failure was persistent or recurrent retinal detachment (10 eyes recorded ophthalmoscopically and 3 by ultrasound). The detachments were due to open retinal breaks (often iatrogenic), were frequently associated with reparative epiretinal 


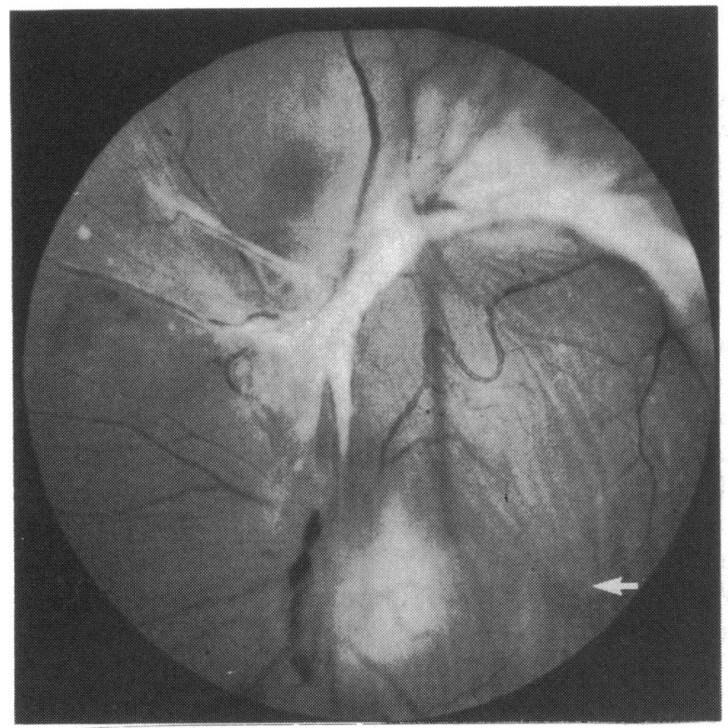

Fig. 3A Eye with grade 1 proliferation preoperatively; extensive combined rhegmatogenous and traction detachment with fovea (arrow) drawn towards disc (acuity 4/60).

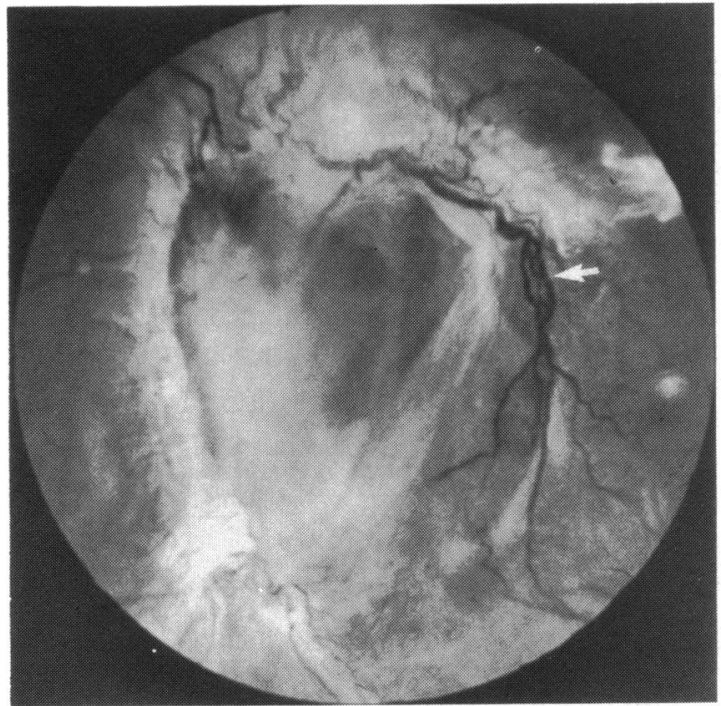

Fig. 3C Eye with grade 3 proliferation preoperatively; traction detachment throughout posterior pole; large vascular channels (arrow) in epiretinal membrane temporal to macula (acuity 2/60).

fibrosis, and in aphakic eyes were invariably complicated by rubeosis iridis, haemorrhage, and phthisis. Vision in eyes with persistent retinal detachment postoperatively was reduced to light perception or no light perception. Causes of failure in eyes with an attached retina included progressive

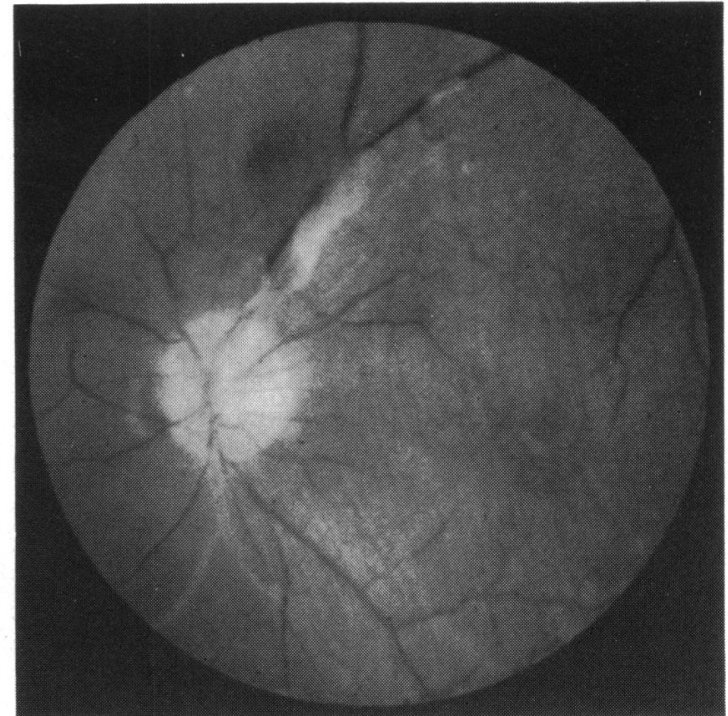

Fig. 3B Same eye as A postoperatively; superotemporal fibrovascular tissue segmented and retina reattached (acuity $6 / 24$.

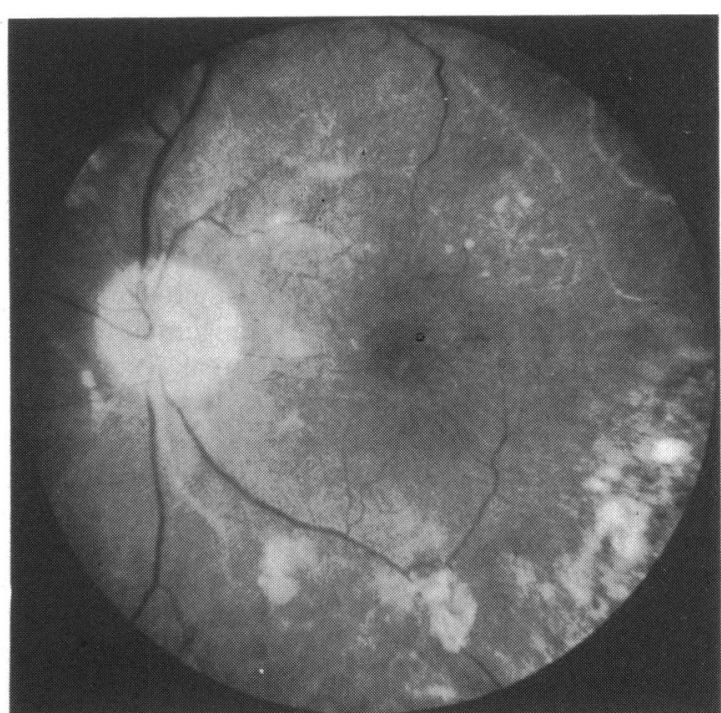

Fig. 3D Same eye and photographic field as $C$ postoperatively; retina reattached with 2 residual segmented outgrowths inferior to macula; intraretinal vasculature attenuated (acuity 6/18).

macular ischaemia, vitreous haemorrhage with or without rubeosis, open-angle glaucoma, and optic atrophy.

An analysis of the anatomical and visual results in relation to the severity grading of the epiretinal vasoproliferation is presented in Fig. 2 and Table 2 . The 
more extensive the membranes, the poorer the rate of retinal reattachment. However, in eyes with successful reattachment of macula the extent of membrane had no influence on the degree of visual improvement (Fig. 2). Examples of successful cases in grades 1 and 3 are illustrated in Fig. 3, which shows fundus photographs of 2 left eyes.

\section{Discussion}

Several authors ${ }^{4-7}$ have presented encouraging results of vitrectomy for macular traction detachment in diabetes, with claims of up to $80 \%$ success. ${ }^{47}$ Comparison with these authors' results is difficult because of differing selection criteria. For example, Aaberg ${ }^{4}$ included only 'pure traction' detachments with no maculopathy and less than 3 months' duration of visual loss. Furthermore, attempts to correlate the results of surgery with such factors as preoperative rubeosis, previous photocoagulation, and lens status have been largely inconclusive, and this has been borne out in our studies. However, we surmised that an important factor influencing surgical success was likely to be the extent of preoperative fibrovascular epiretinal proliferation.

The major technical problem in the surgery of diabetic traction detachment arises during segmentation of coalescent epiretinal membranes. Vitreous scissors are insinuated between the membrane and the retina in areas of 'bridging' or of loose membranoretinal attachment such that individual foci of fibrovascular outgrowth from the retina (where the membranoretinal attachment is most firm) are isolated (Fig. 1D); tangential traction is thereby relieved and associated breaks mobilised. Complications of this manoeuvre include creation of iatrogenic breaks and subsequent reparative (nonvascularised) fibrosis, the latter presumably resulting from retinal surface damage occasioned during the dissection. Problems may also arise from intraoperative bleeding, especially from avulsion of stem new vessels from underlying intraretinal veins; fibrinous clot obscures the surgical field and possibly encourages subsequent fibrosis. ${ }^{2}$ The more difficult and prolonged the dissection the greater the likelihood of such complications. Similarly, repeated introduction of instruments increases the risk of retinal damage at entry sites, though provided this complication is recognised during the operation it is readily treated. The relationship between anatomical success and severity of epiretinal vasoproliferation shown by our results is essentially a reflection of these complications.

In addition to the extent of fibrovascular proliferation its location and configuration may also cause technical difficulties. For example, in eyes with extensive equatorial membranes (and little or no separation between the membranoretinal adhesion and the vitreoretinal attachment at the vitreous base) we generally resort to scleral buckling and cryotherapy rather than prolonged anterior dissection. Similarly, difficulty may be experienced in cases of partial peripheral vitreous detachment when attempts are made to separate the posterior hyaloid from ischaemic atrophic detached retina in order to sever connections between the nonavulsed edge of a posterior fibrovascular membrane and the vitreous base. In eyes with gross contraction of peripapillary membranes (resulting in a tight posterior cone of detachment similar to that seen in massive periretinal proliferation), there are frequently problems in mobilising and reattaching the macula, particularly in view of the difficulty in safely coagulating new vessels arising from the disc circulation.

In those eyes with successful macular reattachment visual improvement is subject to compromise by ischaemic or exudative maculopathy, open-angle glaucoma, rubeosis, or fibrovascular ingrowth and haemorrhage. At the earliest opportunity postoperatively we now carry out (or complete) panretinal argon laser photocoagulation to preempt the development of rubeosis and rebleeding, and we carefully monitor the intraocular pressure. Where such complications can be adequately treated or prevented, visual improvement after macular reattachment is frequently gratifying and bears no relationship to the extent of preoperative fibrovascular proliferation. Final visual acuity is influenced chiefly by the presence of diabetic maculopathy or post-detachment atrophy, the latter determined by the duration and elevation of macular detachment.

We thank Mrs Penny Lovelock for preparing the manuscript and Messrs K. Sehmi and T. R. Tarrant for the figures.

\section{References}

1 Lean JS, Leaver PK, Cooling RJ, McLeod D. The management of complex retinal detachments by vitrectomy and liquid silicone exchange. Trans Ophthalmol Soc UK 1982; 102: 203-5.

2 Charles S. Vitreous microsurgery. Baltimore: Williams and Wilkins, 1981.

3 McLeod D, Leaver PK. Modified Charles flute needle $\mathrm{Br} J$ Ophthalmol 1981; 65: 69 .

4 Aaberg TM. Clinical results in vitrectomy for diabetic traction retinal detachment. Am J Ophthalmol 1979; 88: 246-53.

5 Miller SA. Butler JB, Myers FL, Bresnick GH. Pars plana vitrectomy: treatment for traction macular detachment secondary to proliferative diabetic retinopathy. Arch Ophthalmol 1980; 98: 659-64.

6 McLeod D, Leaver PK, Feretis E. Vitrectomy for severe diabetic eye disease. Trans Ophthalmol Soc UK 1980; 100: 291-8.

7 Charles S. Vitrectomy for retinal detachment. Trans Ophthalmol Soc UK 1980; 100: 542-9. 